\title{
Correction:
}

\section{On Implicit Function Theorems for Set-Valued Maps and Their Application to Mathematical Programming under Inclusion Constraints}

\author{
P. H. Dien and N. D. Yen
}

Institute of Mathematics, P.O. Box 631, Bo Ho, 10,000 Hanoi, Vietnam

Our paper [1] needs the following correction:

The gradient sets $\partial_{x} g_{i}(x, p), i=\widetilde{1, m}$, which appear on pages 45 and 46 should be replaced by the sets

$$
\hat{\partial}_{x} g_{i}(x, p):=\left\{\pi_{1} A: A \in \partial g_{i}(x, p)\right\}, \quad i=\overline{1, m},
$$

where

$$
\left(\pi_{1} A\right)(x):=A(x, 0), \quad \forall x \in X .
$$

The reason for this is that the statement (page 45) saying that the set-valued $\operatorname{map}(x, p) \mapsto \partial_{x} g_{i}(x, p)$ is usc is, in fact, incorrect.

Noting that $\partial_{x} g_{i}(x, p) \subseteq \hat{\partial}_{x} g_{i}(x, p)$ and that the set-valued map $(x, p) \mapsto$ $\hat{\partial}_{x} g_{i}(x, p)$ is usc we see that Corollary 2.2 and the arguments proving it remain true if the above replacements are made.

\section{Reference}

1. Dien PH, Yen ND (1991) On implicit function theorems for set-valued maps and their application to mathematical programming under inclusion constraints. Appl Math Optim 24:35-54 